\title{
Chandrayaan-2 Large Area Soft X-ray Spectrometer
}

\author{
V. Radhakrishna ${ }^{1, *}$, A. Tyagi ${ }^{1}$, S. Narendranath ${ }^{1}$, Koushal Vadodariya ${ }^{1}$, \\ Reena Yadav $^{1}$, Brajpal Singh ${ }^{1}$, G. Balaji ${ }^{1}$, Neeraj Satya ${ }^{1}$, Akash Shetty ${ }^{1}$, \\ H. N. Suresha Kumar ${ }^{1}$, Kumar ${ }^{1}$, S. Vaishali ${ }^{1}$, Netra S. Pillai ${ }^{1}$, S. Tadepalli ${ }^{1}$, \\ Venkata Raghavendra $^{1}$, P. Sreekumar ${ }^{2}$, Anil Agarwal ${ }^{1}$ and N. Valarmathi ${ }^{1}$ \\ ${ }^{1}$ U.R. Rao Satellite Centre, ISRO, Bengaluru 560 017, India \\ ${ }^{2}$ Space Science Programme Office, ISRO Head Quarters, Bengaluru 560 017, India
}

\begin{abstract}
Chandrayaan-2 Large Area Soft X-ray Spectrometer (CLASS) is an X-ray fluorescence spectrometer experiment aimed at mapping the abundances of major rock-forming elements on the lunar surface. The instrument consists of swept charge devices with a passive collimator, visible light blocking filters and signal processing electronics designed and built at U.R. Rao Satellite Centre, Indian Space Research Organisation. CLASS will be the largest collecting area spectrometer flown to the Moon, and thus is expected to map the abundances of lunar elements with a higher sensitivity than ever at soft $X$-ray energies.
\end{abstract}

Keywords: Moon, rock-forming elements, spectrometer, X-ray fluorescence.

THE Moon is considered to have originated from a giant impact with a Mars-sized body during the early years of formation of the solar system. It is a body devoid of any significant atmosphere and hence preserves the rich history of the formation and evolution process of the solar system through its surface features and composition.

Composition of the lunar surface involves identification of elements and minerals present. While minerals reveal the formation history and evolution of the crust, direct detection of elements that are the building blocks of minerals, is critical to decipher degeneracy in mineral signatures and constraining the formation models of the bulk Moon as well as of the various types of lithologies. Also interesting is the inevitable linkage of surface elemental abundances to atoms in the tenuous exosphere. The Moon's surface composition has been determined broadly as consisting of regions of enhanced levels of plagioclase (typical bright highlands) and basalts (typical dark mare regions). However, recent missions with improved spatial and spectral capabilities have revealed a more heterogenic surface with exposures revealing deeper layers and new rock types, whose formation

*For correspondence. (e-mail: rkrish@ursc.gov.in) scenarios challenge current theoretical frameworks. Thus determining elemental abundances is key to constrain lunar origin and evolution models.

X-ray fluorescence spectrometers on Apollo-15 and 16 measured $\mathrm{Mg} / \mathrm{Si}$ and $\mathrm{A} / \mathrm{Si}$ ratios for about $10 \%$ of the Moon on the equatorial region ${ }^{1,2}$. Gamma-ray spectrometers on Lunar Prospector ${ }^{3,4}$ and Kaguya ${ }^{5}$ have mapped the radioactive elements globally along with some of the major elements at resolution of hundreds of kilometres. DCIXS on SMART-1 measured $\mathrm{Ca}$ and $\mathrm{Ti}$ along with $\mathrm{Mg}, \mathrm{Al}$ and $\mathrm{Si}$ on a few regions of the Moon ${ }^{6,7}$. KaguyaXRS, owing to extreme degradation of the detectors, did not function as desired ${ }^{8}$. Chang'E-2 X-ray spectrometer generated global $\mathrm{Mg} / \mathrm{Si}$ and $\mathrm{Al} / \mathrm{Si}$ ratio maps ${ }^{9}$. $\mathrm{C} 1 \mathrm{XS}$ on Chandrayaan-1 measured all the major elements such as $\mathrm{Mg}, \mathrm{Al}, \mathrm{Si}, \mathrm{Ca}$ and $\mathrm{Fe}$ for a few regions ${ }^{10}$ and also $\mathrm{Na}$ for the first time ${ }^{11}$. Due to the limited mission life of the Chandrayaan-1 and low solar activity, global mapping could not be achieved. The Chandrayaan-2 Large Area Soft X-ray Spectrometer (CLASS) experiment ${ }^{12}$, with a spatial resolution of $12.5 \mathrm{~km}$ at its best, is a significant improvement over C1XS, and would be the most sensitive lunar spectrometer to produce surface elemental maps of $\mathrm{Mg}, \mathrm{Al}, \mathrm{Si}, \mathrm{Ca}, \mathrm{Ti}$ and $\mathrm{Fe}$.

As we improve spatial resolution, it is likely that we encounter new compositions as in C1XS, where the presence of $\mathrm{Na}$ at a level of a few per cent by weight was observed. CLASS is thus designed with a larger collection area and higher spatial resolution targeting a quantitative estimate of elemental abundances. Table 1 gives the key features of CLASS payload (shown in Figure 1).

In this article we describe the instrument and its capabilities as derived from ground calibration, relevant to pursue the proposed science goals.

\section{Instrument configuration}

\section{Principle of detection}

Solar X-rays produced during a solar flare impinge on the surface of the Moon and result in fluorescent X-ray emission from the excited atoms. CLASS is designed to 
detect this X-ray emission in the $0.8-15 \mathrm{keV}$ energy range. Since it is important to identify the spectrum of solar flare-produced X-rays that trigger the generation of fluorescent X-rays, a companion payload on Chandrayan2 , the X-ray Solar Monitor ${ }^{13}$, observes the Sun simultaneously to measure solar flare X-ray spectrum.

\section{Detector system}

CLASS consists of an array of 16 swept charge devices (SCDs) operating in the energy range $0.8-15 \mathrm{keV}$ (ref. 14). SCDs are similar to X-ray charge coupled devices (CCDs) except for the readout architecture on the device. CLASS SCDs (CCD-236; make Teledyne e2V), have been flown on the HXMT satellite for astronomical observations ${ }^{15}$. Table 2 gives the device specifications.

Each SCD is built with a total sensitive area of $\sim 4 \mathrm{~cm}^{2}$, internally divided into four sections. In each section, charge is transferred towards the centre of the device, where the charge flows merge before transferring to a single charge-sensitive amplifier. Four SCDs are arranged as a quad, with the four quads forming the full sensitive array of CLASS (Figure 2).

In the absence of true imaging optics, CLASS derives coarse spatial resolution using passive collimators that restrict the instantaneous field-of-view from which photons can enter the detector. CLASS collimators are designed for a ground pixel resolution of $12.5 \mathrm{~km} \times$ $12.5 \mathrm{~km}$ on the lunar surface from a $100 \mathrm{~km}$ altitude orbit, which is an improvement over the C1XS capability $(25 \mathrm{~km} \times 25 \mathrm{~km})$. The collimators made of copper coated with gold, placed above the detectors, make an opening angle of $7.2^{\circ}$, which defines the instantaneous ground pixel of $12.5 \mathrm{~km} \times 12.5 \mathrm{~km}$ on the lunar surface. The SCD effective open area is $84.5 \%$ of its geometrical area.

Table 1. Key features of CLASS

\begin{tabular}{ll}
\hline Energy range & $0.8-15 \mathrm{keV}$ \\
Energy resolution & $<200 \mathrm{eV} @ 5.9 \mathrm{keV}$ \\
IFOV (FWHM) & $12.5 \mathrm{~km} \times 12.5 \mathrm{~km}$ \\
Operating temperature & $-20^{\circ} \mathrm{C}$ (nominal) \\
Power & $27 \mathrm{~W}$ \\
Weight & $15 \mathrm{~kg}$ (includes thermal elements) \\
\hline
\end{tabular}

Table 2. Major specifications of CCD-236 (SCD)

\begin{tabular}{ll}
\hline Active area of unit detector & $\sim 4 \mathrm{~cm}^{2}$ \\
Device technology & Epitaxial, scientific-grade \\
Readout noise & $<10$ electrons at $100 \mathrm{kHz}$ \\
Readout rate & $10-100 \mathrm{kHz}$ \\
Dark signal $\left(@-20^{\circ} \mathrm{C}\right)$ & $<10$ electrons \\
Cycles to clear & $100-130$ \\
Amplifier sensitivity & $>4 \mu \mathrm{V} / \mathrm{e}$ \\
No. of clocking phases & Two-phase \\
Readout type & Continuous clocking \\
\hline
\end{tabular}

In order to block visible light reaching the SCDs, two layers of filters (Luxel Corporation, USA) are used on either side of the collimator. Each filter is a $0.2 \mu \mathrm{m}$ polyimide coated with $0.2 \mu \mathrm{m}$ aluminium bonded on a gold-coated aluminium frame. Gold is coated in order to avoid secondary fluorescence from the aluminium frame.

Each SCD measures the energy of every detected photon which is stored and transmitted to the ground to generate a spectrum of fluorescent X-rays. The events are time-tagged, which gives the time of occurrence of each photon. Thus every spectrum is attached with the time of occurrence and can be correlated to the observed ground pixel on the lunar surface.

\section{Electronics}

The basic elements of CLASS electronics are the following (Figure 3); (i) Front-end electronics, (ii) Digital processing electronics, (iii) Spacecraft interfaces and (iv) Power conditioning and control electronics.

Each SCD constitutes an independent detector with a readout channel yielding a total of 16 channels for the

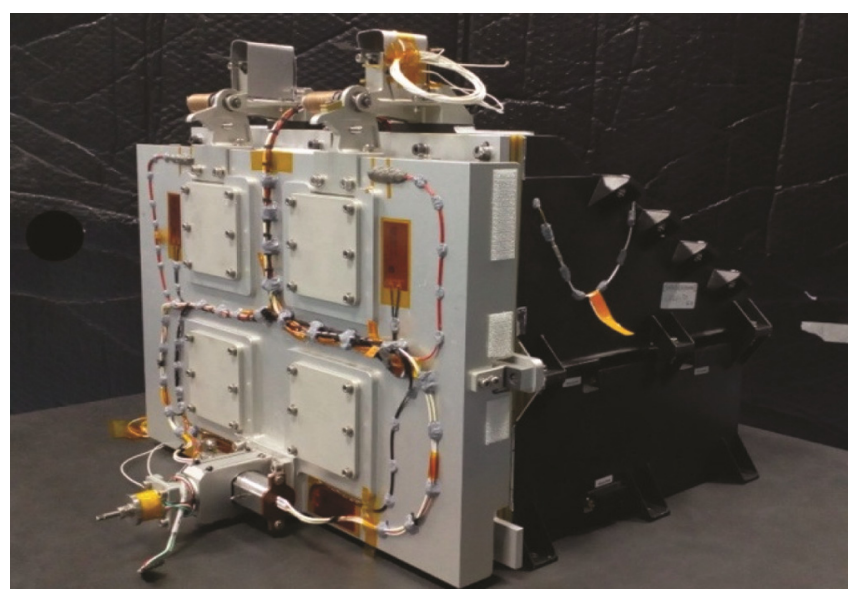

Figure 1. CLASS flight instrument.

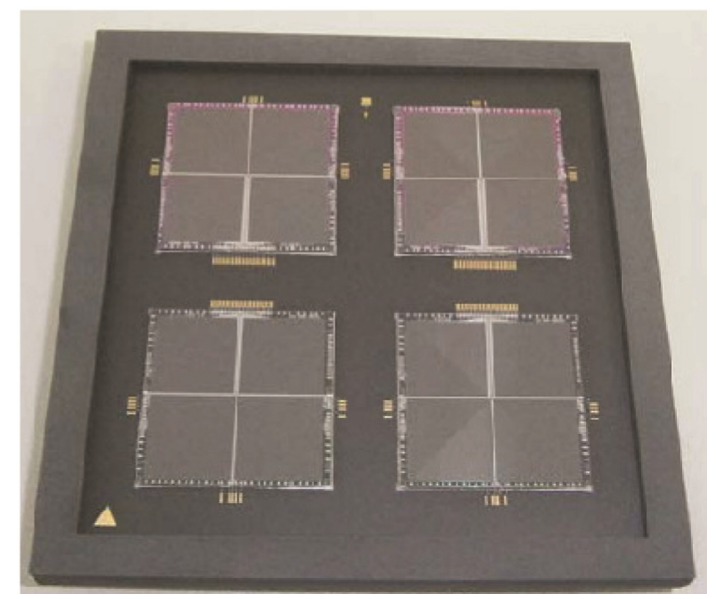

Figure 2. A quad of CLASS with four swept charge devices.

CURRENT SCIENCE, VOL. 118, NO. 2, 25 JANUARY 2020 


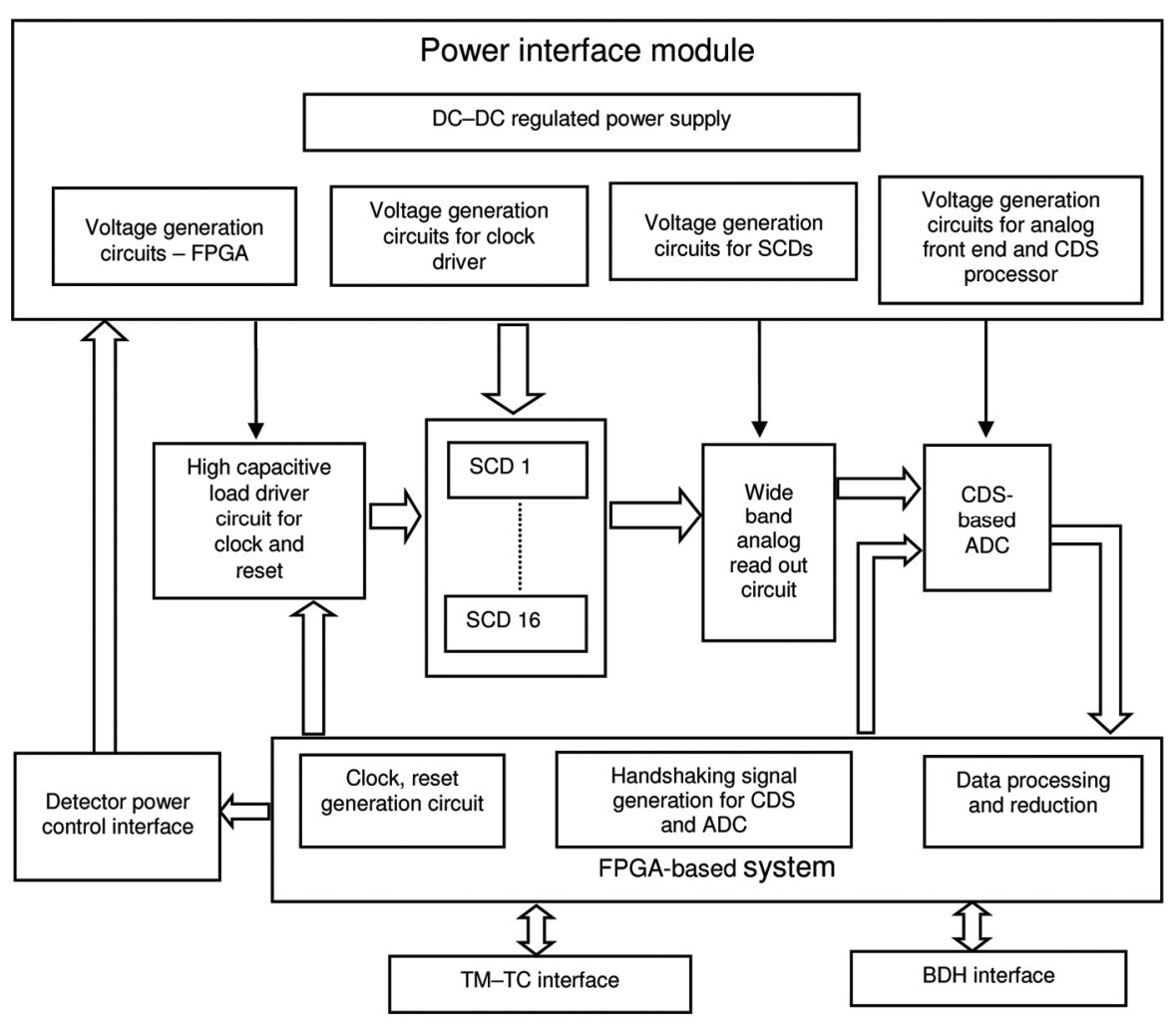

Figure 3. CLASS electronics block diagram.

entire detector system. The device output is connected to the front-end electronics through flexi-rigid lines and a mother board. Front-end electronics consists of a clock driver section which provides two phase readout clocks and two reset clocks.

The low-energy threshold of the detector is $0.8 \mathrm{keV}$ according to the science requirements which would generate a very low signal in CCD-236. Therefore, the signal needs to be amplified before feeding to an analogue to digital converter (ADC). A two-stage amplifier using a high-bandwidth video op-amp (AD8041) is employed to provide a high gain of $\sim 200$. The gain is estimated based on the dynamic range of $0.8-15 \mathrm{keV}$, which corresponds to $110 \mathrm{mV}$ and $4 \mathrm{~V}$ respectively, at the input of the ADC. The ADC (AD981) is three-channel led and has an in-built clamp and sample-based correlated double sampling (CDS), followed by a programmable gain amplifier (PGA) and a digitally controlled offset correction circuit. The SCD read out clock frequency is $100 \mathrm{kHz}$ and hence the AD9814 ADC clock is set at $300 \mathrm{kHz}$. The digital outputs of the ADC are fed to a field programmable gate array (FPGA) for further processing. Each SCD quad module has a thermistor incorporated within the module for measurement of temperature of the device. Temperature and voltage monitoring circuit is incorporated in the front-end card using ADC128S002, a 12-bit ADC.

In digital processing electronics, all the required processing is controlled by RTAX-2000S FPGA. SCDs are continuously clocked and hence all the analogue output signals are continuously digitized in synchronization with the SCD clocks and processed. The 'no signal' or 'zero energy' level is also computed onboard for each SCD and a programmable offset is added to qualify a valid X-ray event. The valid X-ray events are written into the local memory for creation of digital packets. The packets are sent to the base band data handling $(\mathrm{BDH})$ package through the low voltage differential signalling (LVDS) interface. The rate at which packets are sent to the $\mathrm{BDH}$ is decided onboard based on the incoming $\mathrm{X}$-ray event rates and changes from $64 \mathrm{~ms}$ to $4 \mathrm{~s}$. The $64 \mathrm{~ms}$ duration packet can cater up to 32,000 events per second for the total detector area. This logic avoids sending unusable data to the ground and saves onboard storage and transfer bandwidth requirements. The FPGA also monitors the detector temperature onboard and compares it with a programmable pre-defined hightemperature limit for the detector. If the detector temperature crosses this limit, the detector clocks are turned-off.

\section{System design}

The four SCD quad modules are fixed on an aluminium detector base plate with the help of a hold-down fixture. This fixture is designed to provide good mechanical interface with the quad module and detector base plate, and also to accommodate thermal expansions that may occur during changes in temperature in orbit. Electronics packages and detector packages are housed together, yet 
thermally isolated with the GFRP spacer and multilayer insulator (MLI).

\section{Thermal design}

The four quad modules make up an area of about $160 \mathrm{~cm} \times$ $160 \mathrm{~cm}$, dissipating $4 \mathrm{~W}$ power. In addition, there is an additional heat load from the Moon albedo on the device, which varies within an orbit and with seasons. The design is aimed at cooling the devices to $-20^{\circ}$ passively. Two heat pipes connect to a radiator plate which is exposed to the low temperature of deep space $(<4 \mathrm{~K}$; Figure 4$)$.

\section{Radiation shielding}

SCDs are similar in structure to X-ray CCDs and are prone to radiation damage in space. They require shielding from charged particles, without which energy resolution of the spectrometer will degrade with time.

The shielding material and thickness were calculated based on the total dose estimated by SPENVIS (Space Environment Information System $)^{16}$, considering the mission profile and GEANT4 (a Monte-Carlo simulation toolkit) ${ }^{17}$ simulation of the attenuation and secondary radiations. An Al door of $7 \mathrm{~mm}$ thickness (Figure 4) and a $6 \mathrm{~mm}$ thick tantalum block behind the SCDs are incorporated in the configuration for radiation shielding. Tantalum provides radiation shielding throughout the mission life and the door which is closed during the Earth-toMoon transit protects the devices during passage through the radiation belts.

\section{Door mechanism}

There are $16 \mathrm{Fe}-55$ radioactive sources (manufactured at Radio Pharmaceuticals Division, Bhabha Atomic Research

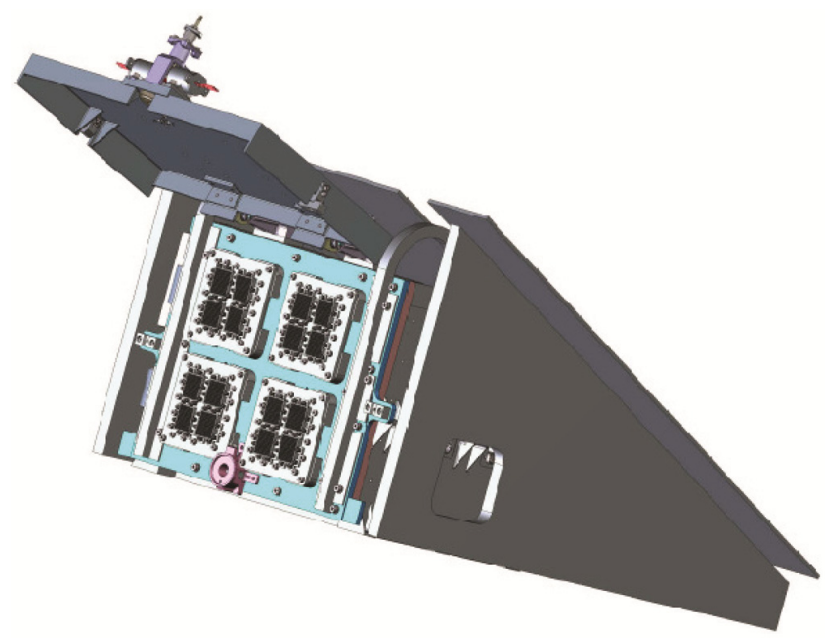

Figure 4. CLASS payload model (includes thermal elements like heat pipe and radiator).
Centre (Mumbai) with a Ti foil mounted on the inside of the CLASS door, one source to shine on each SCD. Before lunar observations begin, the spectra from these sources with four lines $(4.5,4.9,5.9$ and $6.4 \mathrm{keV})$ provide verification of the ground calibration parameters. A few days of calibration phase with the door closed is envisaged for CLASS.

The door opens as lunar observations begin in the nominal orbit. A novel Vectran rope and thermal cutterbased hold-down release mechanism has been employed in CLASS. The Vectran rope is cut on command from the ground by the thermal cutter. A conventional torsion spring-based hinge mechanism is used to deploy the door $110^{\circ}$ from the hold-down plane, clearing the field-of-view of the SCDs. The door is designed for a single operation and will be latched after deployment.

\section{Ground testing and calibration}

The payload has undergone detailed subsystem-level testing, including environmental tests to qualification levels and performance checks. Spectra from Fe-55 sources on the door were recorded and analysed for assessing the performance (Figure 5). During thermo-vacuum tests, detailed calibrations were carried out.

The primary aim of CLASS calibration was to derive the following parameters:

(1) Energy - channel relation as a function of temperature for all SCDs: The X-ray events are binned into 2048 channels which need to be mapped onto energy space to identify the photon energy. This relation is sensitive to the temperature of the device.

(2) Full width at half maximum (FWHM) as a function of energy and temperature for all SCDs: The ability to separate X-ray fluorescence (XRF) lines depends on spectral resolution of the instrument, which can be expressed in terms of FWHM (eV) for the given energy peak. As temperature of the device increases, FWHM broadens and it becomes difficult to separate out closely spaced XRF lines.

(3) Low-energy detection limit of CLASS: One of the targets of CLASS is to measure any possible signatures of the $\mathrm{Na}$ line at $1.04 \mathrm{keV}$. In order to ensure this, the low energy threshold has to be $0.8 \mathrm{keV}$ or lower (accounting for the spread of the peak due to finite FWHM).

The experimental set-up was done in a $1.6 \mathrm{~m}$ diameter vacuum chamber. An X-ray gun mounted on an input port of the vaccum chamber was used to generate the XRF spectrum from a pellet made of JSC-1A (a standard lunar simulant; Figure 6). The XRF emission illuminated all the 16 SCDs simultaneously. Spectra were measured as a function of temperature in steps of about $1{ }^{\circ} \mathrm{C}$.

\section{Data analysis}

The output from the instrument consists of time-tagged events for each SCD, which are binned in time to generate 

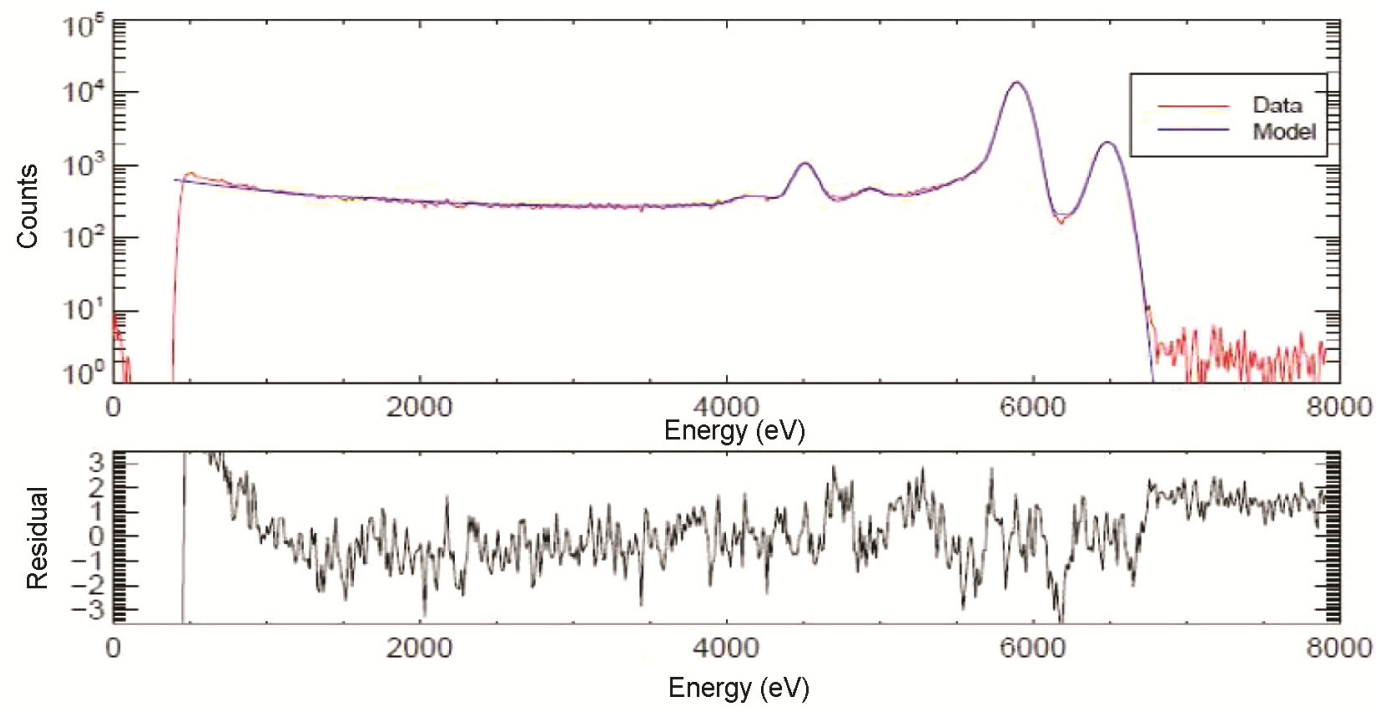

Figure 5. Fit to the Fe-55 onboard source spectrum of the combined SCDS with Gaussian lines for the peaks, a low energy tail and shelf.

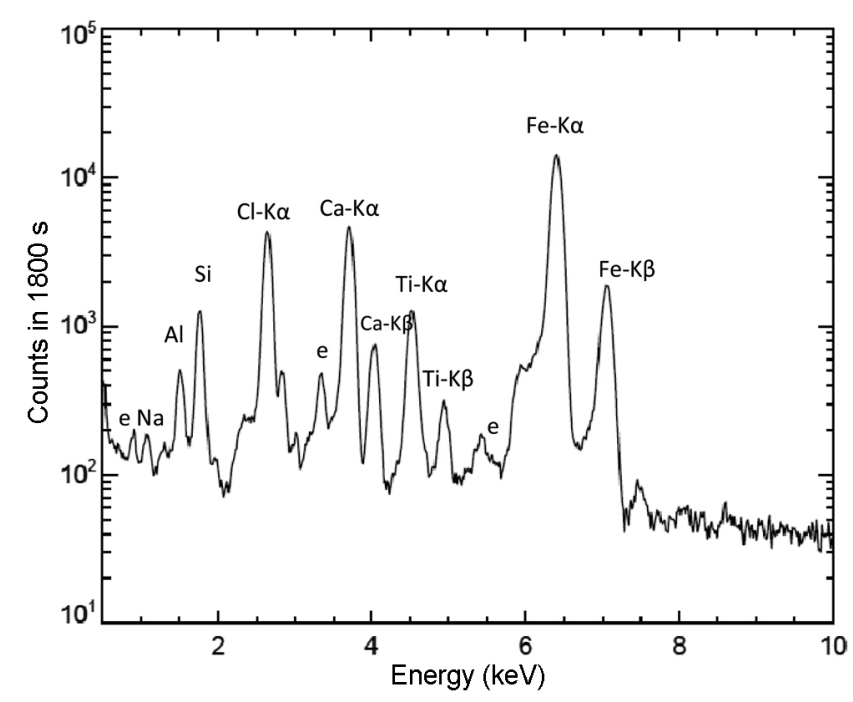

Figure 6. JSC-1A with $\mathrm{NaCl}$ spectrum in CLASS demonstrating the low energy threshold. 'e' represents escape peaks.

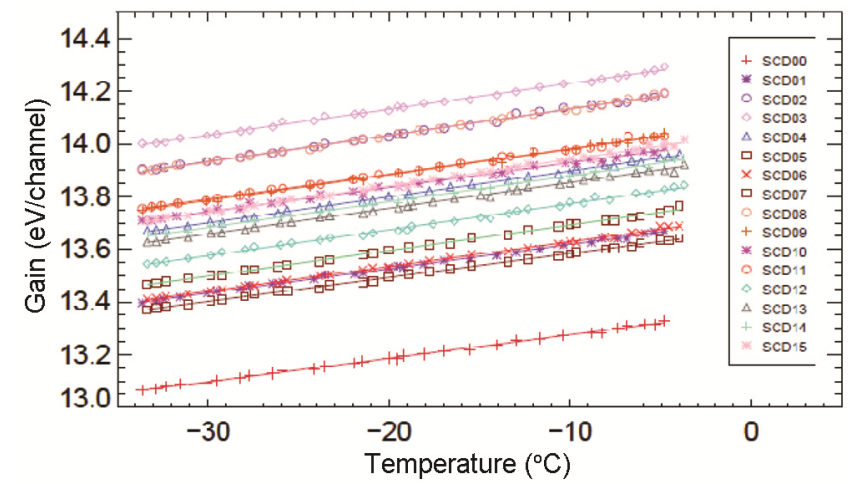

Figure 7. Change in gain with temperature for the $16 \mathrm{SCDs}$ is linear with an average slope of $0.0096 \mathrm{eV} / \mathrm{channel} /$ degree. The gain correction is applied to each of the SCD spectra before adding them to generate level-1 data. the spectra. The spectral lines were fit using a Gaussian function and 'mpfit' routine in IDL, and the calibration parameters were derived for all 16 devices.

\section{Results}

Figures 7 and 8 plot the gain and FWHM of the instrument as a function of temperature. At the nominal operating temperature of $-20^{\circ} \mathrm{C}, \mathrm{FWHM}$ of all the devices is less than $200 \mathrm{eV}$ at $5.9 \mathrm{keV}$. The FWHM derived from the summed spectra of all the $16 \mathrm{SCDs}$ is $165.9 \pm 0.5 \mathrm{eV}$ at $-20^{\circ} \mathrm{C}$.

In order to demonstrate the low energy threshold, common salt $(\mathrm{NaCl})$ was added to the JSC-1 A target and data taken in an experimental configuration similar to that described above. Single events were selected from the $1800 \mathrm{sec}$ long integrated spectrum at $-20^{\circ} \mathrm{C}$. The line from $\mathrm{Na}(1.04 \mathrm{keV})$ can be clearly seen (Figure 6), thus demonstrating the low energy performance.

The effective area of the instrument is defined as:

Effective area $=$ geometric area $\times$ detection efficiency $(E) \times$ filter transmission $(E) \times$ collimator open area fraction.

The energy-dependent detection efficiencies are calculated from the mass attenuation coefficient provided by NIST-XCOM (https://physics.nist.gov) in the energy range above $2 \mathrm{keV}$. Below $2 \mathrm{keV}$, measurements at the synchrotron facility in Physikalisch-Technische Bundesanstalt (PTB), Germany by a team from the Open University, UK (Jason Gow, private commun.) are used. The open area fraction of the collimators was measured with a beam of visible light in the laboratory. 


\section{RESEARCH ARTICLES}

Figure 9 shows the resulting effective area estimation for CLASS.

In order to extract the flux (photons $/ \mathrm{cm}^{2}$ ) under an observed XRF line, the response of the instrument for a given incident energy must be well understood. From the ground calibration parameters and effective area, we have

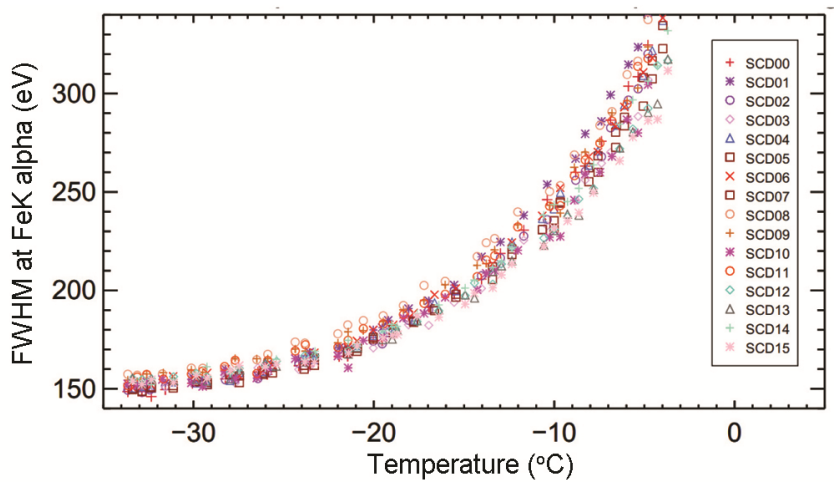

Figure 8. Change in FWHM with temperature. The noise factor in terms of the number of the electrons is computed and incorporated in the response matrix at the operating temperature.

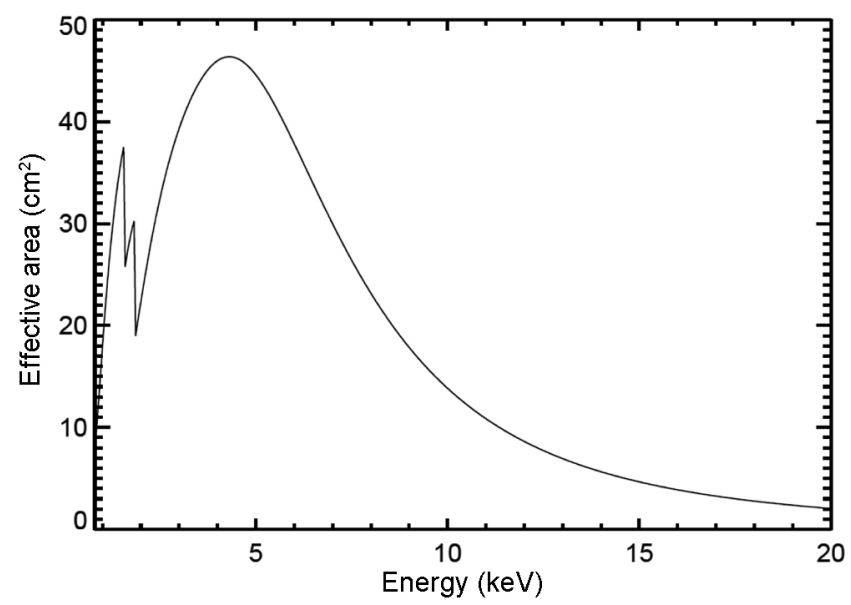

Figure 9. CLASS effective area where the detection efficiency of SCD in the $0.8-2 \mathrm{keV}$ range is measured at the BESSY II synchrotron facility. The rest of the curve is calculated.

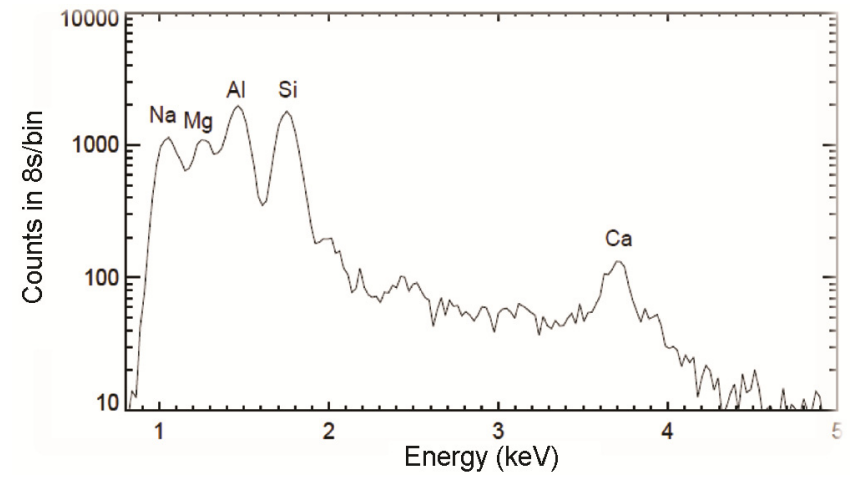

Figure 10. Expected spectrum in CLASS for a B-class solar flare along with background. constructed response matrices (a matrix that contains information for convolving the incident spectrum to what is observed in the detectors) compatible with the standard $\mathrm{X}$-ray analysis package, XSPEC ${ }^{18}$. Figure 10 shows a simulated spectrum in CLASS during a B-class solar flare. The signals are discernible even during the lowintensity flare, providing confidence on the performance of CLASS in the lunar orbit.

\section{X-ray line flux to abundances}

The spectrum measured in the lunar orbit will consist of a continuum component and contribution from X-ray fluorescence lines. Low-energy electrons in the range of tens of $\mathrm{keV}$, deposit their energy in the CLASS detectors, which appears as a continuum emission. During flares the scattered solar flux also adds to this component.

The X-ray line flux is extracted by modelling the observed spectrum as arising from a combination of the scattered solar spectrum and XRF lines. The background arising from the particles is derived from pre-flare spectra and subtracted.

We have developed an algorithm named x2abund based on the fundamental parameter method ${ }^{19,20}$, which has been verified with several experiments in the laboratory. It has also been used to determine elemental maps from C1XS data.

The algorithm calculates scattering from the incident solar spectrum which is then fed into the analysis package (XSPEC) as a model. The extracted XRF line flux is then compared to the flux calculated from $x 2$ abund. The uniqueness of the approach lies in the fact that we use a matrix of elements with a wide range in compositions expressed as weight percentage without any preassumptions on the composition. The best fit abundances are estimated by the method of weighted least squares.

\section{CLASS data products}

The high-level data products from CLASS include the following:

- Combined $8 \mathrm{~s}$ spectrum of all the 16 detectors in FITS format with response matrices (level 1).

- X-ray line flux from observed elements in the lunar XRF spectrum with lunar coordinates (level 2).

- Elemental maps with \% weight as per coverage (level 3).

The CLASS data will be archived in PDS4 standard and made available to public after a lock-in period of 12 months.

1. Adler, I. et al., Apollo 15 and 16 results of the integrated geochemical experiment. Moon, 1973, 7, 487-504.

CURRENT SCIENCE, VOL. 118, NO. 2, 25 JANUARY 2020 
2. Clarke, P. E. and Hawke, B. R., The lunar farside: the nature of the highlands east of Mare smythii. Earth, Moon Planets, 1991, 53, 93-107.

3. Lawrence, D. J., Feldman, W. C., Barraclough, B. L. and Binder, A. B., Global elemental maps of the Moon: the Lunar Prospector Gamma Ray Spectrometer. Science, 1998, 281, 14841489.

4. Prettyman, T. H., Jagerty, J. J., Elphic, R. C., Feldman, W. C., Lawrence, D. J., Mckinney, M. K. and Vaniman, D. T., Elemental composition of the lunar surface: analysis of gamma ray spectroscopy data from Lunar Prospector. JGR, 2006, 111, E12007 (1-41).

5. Hasebe, N., Yamashita, N., Karouji, Y., Kobayashi, S., Hareyama, M., Komatsu, S. and Kim, K. J., Lunar gamma-ray observation by Kaguya GRS, 2010. In Advances in Geosciences, 2010, Volume 19: Planetary Science, pp. 57-67.

6. Grande, M. et al., The D-CIXS X-ray spectrometer on the SMART-1 mission to the Moon - first results. Planet. Space Sci., 55, 494-502.

7. Swinyard, B. M. et al., X-ray fluorescence observations of the Moon by SMART-1/D-CIXS and the first detection of Ti-Ka from the lunar surface. Planet. Space Sci., 2009, 57, 744-750.

8. Okada, T. et al., X-ray fluorescence experiments on the SELENE (KAGUYA) spacecraft. 2008, In 39th Lunar and Planetary Science Conference Abstr., 1966.

9. Dong, W.-D., Zhang, X., Zhu, M.-H., Xu, A. A. and Tang, Z. S., Global $\mathrm{Mg} / \mathrm{Si}$ and $\mathrm{Al} / \mathrm{Si}$ distributions on lunar surface derived from Chang E-2 X-ray spectrometer. Res. Astron. Astrophys., 2015, 16; 10.1088/1674-4527/16/1/004.

10. Narendranath, S. et al., Lunar X ray fluorescence observations by the Chandrayaan 1 X-ray spectrometer $(\mathrm{C} 1 \mathrm{XS})$ : results from the nearside southern highlands. Icarus, 2011, 214, 53-66.

11. Athiray, P. S., Narendranath, S., Sreekumar, P. and Grande, M., C1XS results - first measurement of enhanced sodium on the lunar surface, PSS, 2014, 104, 279-287.
12. Radhakrishna, V. et al., The Chandrayaan-2 Large area X-ray Spectrometer. In Lunar and Planetary Science Conference, abstr. no. 1708,2011

13. Shanmugam, M., Vadawale, S., Acharya, Y. B., Goyal, S. K., Patel, A., Shah, B. and Murthy, S. V. S., The solar X-ray monitor on Chandrayaan-2. In 43rd LPSC, abstr. no. 1858, 2012.

14. Lowe, B. G., Holland, A. D., Hutchinson, I. B., Burt, D. J. and Pool, P. J., The Swept Charge Devices, a novel CCD - Based EDX detector: first results, NIM A, 2001, 458, 568-579.

15. Wu, Y. P., Dahai, R. and You, Z., HXMT satellite for space hard X-ray observations. Adv. Space. Res., 2004, 34, 2667-2672.

16. Space Environment Information System; http://spenvis.oma.be.

17. Agostinelli, S. et al., Geant4: a simulation Toolkit. Nucl. Instrum. Methods A, 2003, 506, 250-303.

18. Arnaud, K. A., Astronomical data analysis software and systems. Astron. Softw. Proc., 1996, 101, 17-20.

19. Athiray, P. S. et al., Experimental validation of XRF inversion code for Chandrayaan-1. Planet. Space Sci., 2013, 89, 183-187.

20. Athiray, P. S., Narendranath, S., Sreekumar, P., Dash, S. K. and Babu, B. R. S., Validation of methodology to derive elemental abundances from X-ray observations on Chandrayaan-1. Planet. Space Sci., 2013, 75, 188-194.

ACKNOWLEDGEMENTS. We thank Dr Usha Pandey and her team at the Radio pharmaceuticals Division, Bhabha Atomic Research Centre for providing radioactive sources for onboard calibration; Dr Jason Gow and his colleagues at Open University, UK; Mr Chris Howe at Rutherford Appleton Laboratory, UK; Mr C. V. Sreekantha (URSC, ISRO) for help during the configuration of the payload, and $\mathrm{Mr}$ Gaurav Sharma and Mr Vishal Nagarakoti (SMG, URSC, ISRO) for mechanism analysis and hardware testing support respectively.

Received and accepted 27 August 2019

doi: $10.18520 / \mathrm{cs} / \mathrm{v} 118 / \mathrm{i} 2 / 219-225$ 